ScIDice

\section{Comparison Of Simplified Autologous Fibrin Glue Versus Suturing For Periodontal Flap Closure - A Randomised Controlled Trial}

Research Article

Subasree Soundarajan ${ }^{1 *}$, Arvina Rajasekar ${ }^{2}$

${ }^{1}$ Department of Periodontics, Saveetha Dental College and Hospitals, Saveetha Institute of Medical and Technical Sciences, Chennai-600077, Tamil Nadu, India.

${ }^{2}$ Senior Lecturer, Department of Periodontics, Saveetha Dental College and Hospitals, Saveetha Institute of Medical and Technical Sciences, Chennai-600077, Tamil Nadu, India.

\title{
Abstract
}

Objective: to assess the post operative healing and stability of flap closure using autologous fibrin glue when compared to silk suture.

Methodology: Thirty subjects with moderate to severe periodontitis who requires periodontal flap surgery involving at least four adjacent teeth, as determined by clinical criteria (i.e., probing depth of $5 \mathrm{~mm}$ and bone loss on radiograph of $50 \%$ ), between 20 and 50 years of age of both sexes, and showing adequate oral hygiene before surgical therapy were included in the study. Autologous fibrin glue was prepared. The surgical area to be treated was randomly selected into two areas, Group A: Two papilla were secured with sutures (3-0 silk) after surgery; Group B: Two papilla were secured with autologous fibrin glue after surgery.

Parameters assessed \& tests performed - The roll test for flap closure stability was used to verify the flap's adhesion. The postoperative healing was evaluated using the simplified healing index.

Results: In the inter group comparisons of Simplified healing index, there was a statistically significant difference between two study groups (Silk suture versus Autologous fibrin glue) at 1 week follow up with a better healing in autologous fibrin glue group with a $\mathrm{p}$ value of 0.003 . Whereas, the inter group comparisons of Roll test score at 1 week follow up, there was no significant difference between two study groups (Silk suture versus Autologous fibrin glue) with a p value of 0.1. Hence, both autologous fibrin glue and silk suture showed similar flp adaptation 1 week after flap surgery. In the intra group comparisons, significant difference was seen in Roll test score between baseline and 1 week follow up with a p value $<0.01$ in both the groups (Silk suture versus Autologous fibrin glue).

Conclusion: This autologous fibrin glue preparation is not only quick and easy to prepare, but it also offers excellent postoperative healing and flap closure stability that is comparable to or better than silk sutures. It is also less traumatic, quicker at the chairside, and needs less skills to use than silk sutures.

\section{Introduction}

Suturing is an important aspect of periodontal flap surgery since it is used in flap closure and is crucial to its performance. Sutures, on the other hand, have drawbacks such as plaque management issues, increased postoperative pain, increased tissue reactivity, and a higher infection risk [1]. These limitations have prompted researchers to look for alternative approaches, including sutureless techniques. Fibrin sealant, also known as fibrin glue, has been used as a sealant and for hemostasis in surgery [2]. It is a biological tissue adhesive that mimics the final stages of coagulation. It has been used to secure gingival grafts and mucoperiosteal flaps [3]. Fibrin sealant can be used for wound closure replacing the need for sutures, even in locations and circumstances where suturing would be detrimental to optimal wound healing and integrity $[4$, 5]. It has potential adhesive properties that effectively seal the tissues and eliminate the dead spaces. Apart from binding tissues, fibrin sealant also acts as a natural wound bed. This bed acts as a scaffold, allowing mesenchymal and endothelial cells to proliferate and differentiate more easily $[6,7]$. Improved plaque control,

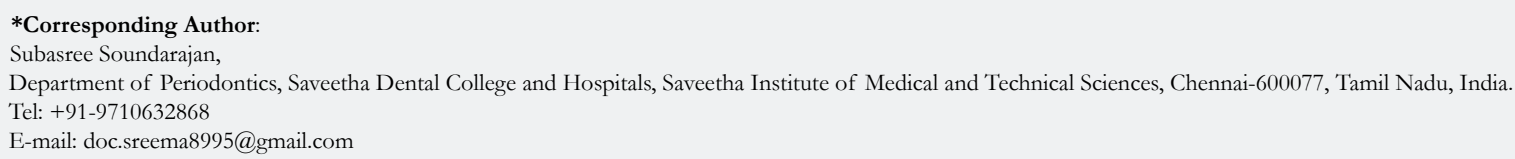

Citation: Subasree Soundarajan, Arvina Rajasekar. Comparison Of Simplified Autologous Fibrin Glue Versus Suturing For Periodontal Flap Closure - A Randomised Controlled Trial. Int J Dentistry Oral Sci. 2021;8(8):3810-3815. doi: http://dx.doi.org/10.19070/2377-8075-21000781

Copyright: Subasree Soundarajan ${ }^{\circ} 2021$. This is an open-access article distributed under the terms of the Creative Commons Attribution License, which permits unrestricted use, distribution and reproduction in any medium, provided the original author and source are credited. 
less postoperative pain, lower infection rates, and quicker healing are few of the benefits of autologous fibrin glue. When compared to sutures, it has benefits such as better plaque control, less postoperative pain, lower infection rates, faster healing, no special expertise required for application, and improved aesthetics (4). It is atraumatic and less time-consuming with better postoperative healing [8]. It is even been used to keep the free gingival graft immobilised [9].

In comparison to sutures, fibrin sealant proves to be a good alternative for tissue fixation. Since fibrin sealant can seal the entire undersurface rather than only the marginal fixation possible with sutures, the fixation is stronger [10]. It helps in faster wound healing since the patient's oral hygiene maintenance is found to be better when compared to suturing. There were less clinical symptoms of inflammation. The grafts and flaps were more secure, and also shows faster healing by 1 or 2 weeks after surgery [11]. By accelerating revascularization and promoting fibroblast migration, fibrin sealants promote early wound healing and connective tissue development. The sealant is biocompatible and causes only a minor inflammatory response. It can promote angiogenesis, create a more robust epithelium-connective tissue interface, and make connective tissue more resistant to proteolytic enzymes [12]. After 1 week of healing after fibrin sealant application, there is more mature epithelium, connective tissue, increased density of fibroblasts, and mature collagen, while the sutured site has more inflammatory cells [13].

Fibrin glue is widely available or can be made using autologous methods. Both have drawbacks, and hence are not commonly used. Increased cost, multiple components (fibrinogen, thrombin, afibrinolytics, fibronectin, and calcium chloride) involved in preparation, short shelf life, not always available on demand, chances of allergic reaction to bovine protein, and transmission of viral infection are all limitations for commercial products like Tisseel and Reliseal [14]. Whereas, the Autologous preparations also pose a challenge. This includes obtaining the materials required for the preparation, several components are involved (fibrinogen, calcium chloride, thrombin, and afibrinolytics), temperature sensitive preparation procedure, complex, and time consuming chairside methodology [15].

Fibrin sealants are becoming more common in the surgical field as a result of the numerous complications caused by sutures [16]. It is a multi-purpose surgical glue that can be used as a hemostat, sealant, or adhesive to improve the overall clinical outcome of surgical procedures. Several authors have verified the product's efficacy while also demonstrating the product's excellent local tolerability and total lack of side effects and contraindications [17]. It also aids in the healing of periodontal wounds. However, there are contradictory reports in the literature, and further research is required to determine the most appropriate use of these biologics. To the best of the authors' knowledge, the present study is first of its kind to assess the post operative healing and stability of flap closure using autologous fibrin glue when compared to silk suture.

\section{Materials and Method}

\section{Study participants}

Thirty patients were selected in total. Patients with moderate to severe periodontitis who requires periodontal flap surgery involving at least four adjacent teeth, as determined by clinical criteria (i.e., probing depth of $5 \mathrm{~mm}$ and bone loss on radiograph of $50 \%$ ), between 20 and 50 years of age of both sexes, and showing adequate oral hygiene before surgical therapy were included in the study. Patients with any known systemic diseases and/or drug therapy that may interfere with wound healing, drug allergies to any of the drugs used in the study, smoking, tobacco chewing, or any other habit that may affect the disease or treatment, and pregnant or lactating mothers were excluded from the study. The research was performed in accordance with the Helsinki Declaration of 1975, as amended in 2013, and the protocol was approved by the institutional review committee for human subjects.

\section{Surgical procedure}

All the participants received Phase 1 therapy and oral hygiene instructions. Subjects were reviewed four weeks after the end of phase one therapy and patients whose inflammation had subsided were scheduled for surgery. The surgical procedure was performed using routine local anesthesia ( $2 \%$ lignocaine with epinephrine 1:100,000) and sulcular incision was made. On the buccal aspects of teeth, the conventional nondisplaced mucoperiosteal flap was elevated. Thorough debridement was carried out to remove the local irritants and diseased granulation. The flap was trimmed and repositioned after debridement to achieve as much total interproximal closure as possible.

The surgical area to be treated was randomly selected into two areas as Group A and Group B.

Group A: Two papilla were secured with sutures (3-0 silk) after surgery.

Group B: Two papilla were secured with autologous fibrin glue after surgery.

Method of preparation and application of autologous fibrin glue [18]

For autologous fibrin glue preparation, $10 \mathrm{~mL}$ of patient blood was collected in sterile 0.9 percent sodium citrate containing vacutainers and centrifuged at $3000 \mathrm{rpm}$ for 10 minutes. This vacutainer now had a layer of platelet-poor plasma on top, a layer of platelet-rich plasma in the centre, and a layer of red blood cells at the bottom (RBCs). The platelet-poor and platelet-rich plasma is drawn into a sterile syringe and kept in a separate test tube without anticoagulant. The RBC fraction was thrown away. To precipitate the most amount of fibrinogen, protamine sulphate $(10 \mathrm{mg} / \mathrm{ml})$ was applied to platelet-rich and platelet-poor plasma. This test tube was centrifuged for 5 minutes at $1000 \mathrm{rpm}$. A top layer of serum with thrombin (autologous) and a bottom layer of fibrinogen precipitate were present in the centrifuged tube.

The top serum was removed, but $0.5 \mathrm{~mL}$ of it was retained in the test tube to dilute the fibrinogen precipitate, and aspirated into a separate syringe. A second syringe was filled with calcium chloride $(0.025 \mathrm{mmol} / \mathrm{l})$, and both solutions were applied in similar amounts under the flaps, which were held under digital pressure for 2-3 minutes.Since the agents used here were obtained from the subject, there are no risks of adverse effects. (Figure 1-7) 
Figure 1. Components for preparation of fibrin glue.

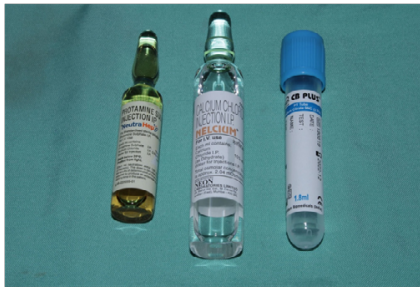

Figure 2. Platelet - rich plasma + platelet poor plasma separated after first centrifugation.

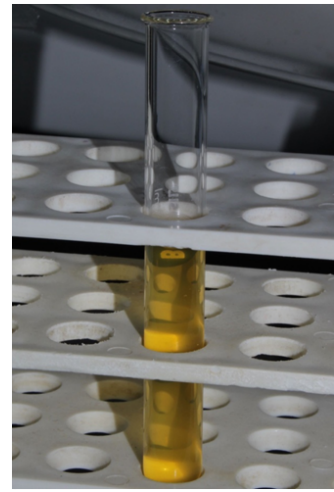

Figure 3. Fibrinogen precipitate sediment after second centrifugation.

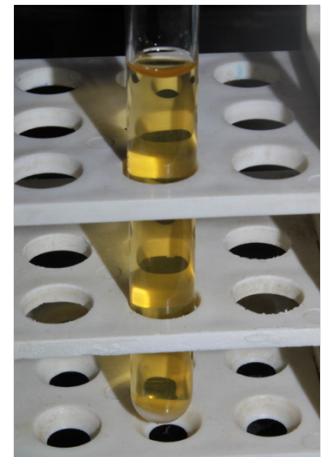

Figure 4. Pre operative.

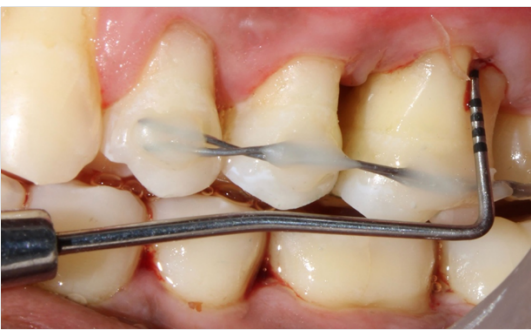

Figure 5. Intra operative.

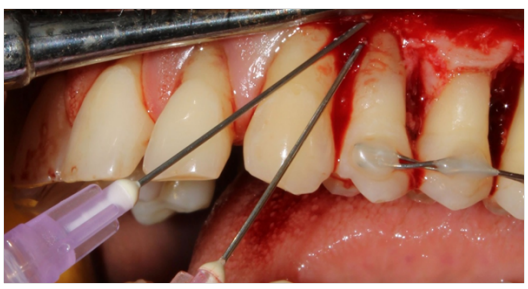

Parameters assessed \& tests performed

1. Roll test [18] - The roll test for flap closure stability was used to verify the flap's adhesion. It is performed by placing the side of the periodontal probe at the base of each papilla, roll the probe in an apical direction, and evaluate the movement of the papilla. Criteria for evaluation is given in Table 1

2. Simplified healing index - The postoperative healing was evalu- ated using the simplified healing index, Sunil and Bhumit-2016 [18], a modification of the healing index given by Landry in 1988 [19] The index was simplified to make it easier to score due to the subjective difficulty of evaluation. as given in Table 2 .

\section{Statistically Analysis}

Chi square tests (inter group comparison) were performed to as- 
Figure 6. Post operative.

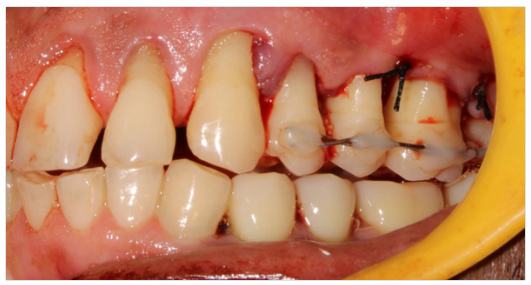

Figure 7. 1 week follow up.

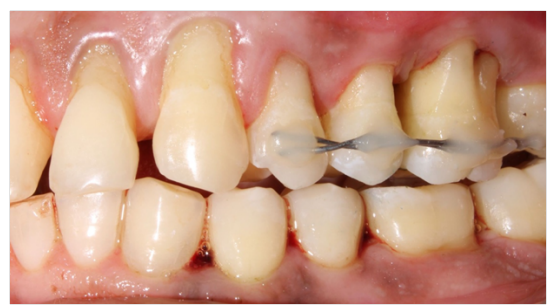

Figure 8. Bar graph depicting the comparison of Simplified healing index at 1 week post op between two groups. $\mathrm{X}$ axis represents Simplified healing index and $\mathrm{Y}$ axis represents study groups (Silk suture \& Fibrin glue). Blue denotes Score 1 - Good healing, green denotes Score 2 - Fair healing and orange denotes score 3 - Poor healing. Chi square test was done and association between simplified healing index at 1 week and type of intervention was found to be statistically significant (Pearson's Chi square value : 11.6, df - 2, p value - 0.003 - statistically significant). Therefore, autologous fibrin glue showed better healing at 1 week following periodontal flap surgery when compared to silk sutures.

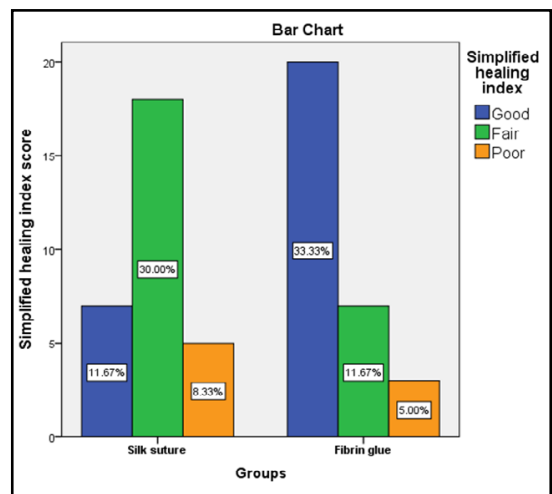

Figure 9. Bar graph depicting the comparison of Roll test score at 1 week post op between two groups. $\mathrm{X}$ axis represents Roll test score and Y axis represents study groups (Silk suture \& Fibrin glue). Red denotes Score 1 - Barely visible movement of the papilla and blue denotes Score 2 - Clearly visible movement but no retraction of the papilla. Chi square test was done and association between Roll test score at 1 week and type of intervention was not statistically significant with a $p$ value - 0.1. Therefore, autologous fibrin glue and silk suture showed similar flap adaptation at 1 week following periodontal flap surgery.

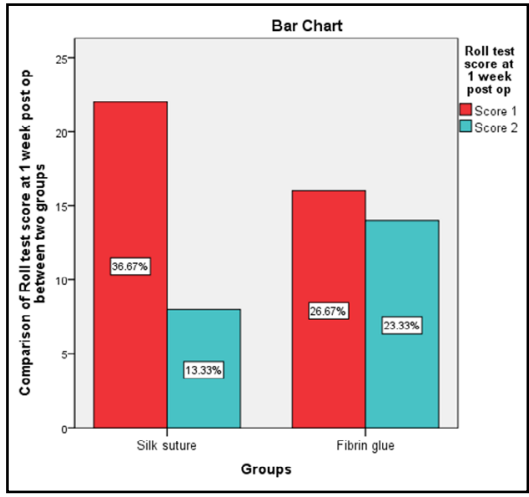

sess the association between a) Roll test score at 1 week post operatively and type of flap closure (Autologous fibrin glue versus silk suture) \& b) Simplified healing index at 1 week post operatively and type of flap closure (Autologous fibrin glue versus silk suture). Paired t test was performed to compare the Roll test score between baseline and 1 week post operative for both the groups (Intra group comparison). All statistical analyses were performed using SPSS version 23.0 (Statistical Package for the social sciences). $\mathrm{P}<0.05$ was considered as statistically significant.

\section{Results}

In the inter group comparisons of Simplified healing index, there 
Table 1. Criteria for Roll test.

\begin{tabular}{|c|c|}
\hline Score & Criteria \\
\hline Score 1 & Barely visible movement of the papilla \\
\hline Score 2 & $\begin{array}{c}\text { Clearly visible movement but no re- } \\
\text { traction of the papilla }\end{array}$ \\
\hline Score 3 & $\begin{array}{c}\text { Clearly visible movement with retrac- } \\
\text { tion of the papilla }\end{array}$ \\
\hline
\end{tabular}

Table 2. Simplified healing index.

\begin{tabular}{|c|c|}
\hline Score & Criteria \\
\hline \multirow{4}{*}{ Score 1 - GOOD } & - Tissue color : All tissue pink \\
\hline & - $\quad$ Response to palpation : No bleeding \\
\hline & - Granulation tissue : None \\
\hline & - Incision margin : No connective tissue exposed \\
\hline \multirow{4}{*}{ Score 2 - FAIR } & Tissue color $:<50 \%$ of gingiva - red \\
\hline & - $\quad$ Response to palpation : No bleeding \\
\hline & - Granulation tissue : None \\
\hline & Incision margin : No connective tissue exposed \\
\hline \multirow{4}{*}{ Score 3 - POOR } & - $\quad$ Tissue color $: \geq 50 \%$ of gingiva red \\
\hline & - $\quad$ Response to palpation : Bleeding \\
\hline & - $\quad$ Granulation tissue : Present \\
\hline & $\begin{array}{l}\text { Incision margin : Not epithelialized, with loss of epithelium } \\
\text { beyond incision margin; Suppuration might be present. }\end{array}$ \\
\hline
\end{tabular}

was a statistically significant difference between two study groups (Silk suture versus Autologous fibrin glue) at 1 week follow up with a better healing in autologous fibrin glue group with a $\mathrm{p}$ value of 0.003 (Figure 8).

Whereas, the inter group comparisons of Roll test score at 1 week follow up, there was no significant difference between two study groups (Silk suture versus Autologous fibrin glue) with a $\mathrm{p}$ value of 0.1 (Figure 9). Hence, both autologous fibrin glue and silk suture showed similar flp adaptation 1 week after flap surgery.

In the intra group comparisons, significant difference was seen in Roll test score between baseline and 1 week follow up with a $\mathrm{p}$ value $<0.01$ in both the groups (Silk suture versus Autologous fibrin glue).

\section{Discussion}

In these cases, we attempted to assess the efficacy of flap closure with autologous fibrin glue and silk sutures. The use of autologous fibrin glue, prepared using a simplified and new method (a combination and modification of Hartman's and Altson's methods), is the focus of this study [14]. The findings showed that areas closed with fibrin glue healed faster than areas closed with sutures, similar to the results obtained by Bimal et al. using Tisseel [2]. The healing index outperformed Landry et al's [7] index because it has clearer ratings, is easier to classify, eliminates subjective assessment complexity, and is more focused on periodontal healing. The roll test used in this study has been used in a case report by Dave et al. A study done by Pini Prato et al. using Tissuecol [8] found that fibrin glue was also less time-consuming and simple to apply during chairside use.

Other tissue adhesives, such as cyanoacrylate (synthetic in nature), have been abandoned due to high tissue toxicity, and fibrin glue tends to be a safer option. [8] Traditional sutures only offer a marginal fixation, while fibrin glue adheres the tissues to the entire surface [2]. The use of sutures and fibrin glue in the same quadrant rather than separate quadrants is one of the drawbacks of our study. Using fibrin glue to close the flap is simpler and faster, but additional preparation time away from the chairside in the laboratory, the use of equipment such as a centrifuge, and materials for preparing the flap are required.

Despite a few limitations, the current study demonstrates the successful use of a simplified fibrin glue preparation that not only had all of the benefits of previous fibrin glue preparations, but also had the added benefits of a lower cost and a simpler procedure for preparation under normal chairside/laboratory conditions, without the use of complicated techniques or sophisticated procedures. Flap closure using fibrin glue showed better and faster healing with reduced inflammation clinically. In terms of flap adaptability, both silk suture and fibrin glue show similar results. Thus, autologous fibrin seems to be promising and it could be used as an alternative to suturing, thereby marking the beginning of a sutureless era [7].

\section{Conclusion}

This autologous fibrin glue preparation is not only quick and easy to prepare, but it also offers excellent postoperative healing and flap closure stability that is comparable to or better than silk su- 
tures. It is also less traumatic, quicker at the chairside, and needs less skills to use than silk sutures.

\section{References}

[1]. Pulikkotil SJ, Nath S. Fibrin sealant as an alternative for sutures in periodontal surgery. J Coll Physicians Surg Pak. 2013 Feb 1;23(2):164-5.

[2]. Bartolucci EG, Prato GP. Preliminary observations on the use of a biologic sealing system (Tissucol) in periodontal surgery. J Periodontol. 1982 Dec;53(12):731-5.Pubmed PMID: 6984067.

[3]. Pini Prato GP, Cortellini P, Agudio G, Clauser C. Human fibrin glue versus sutures in periodontal surgery. J Periodontol. 1987 Jun;58(6):426-31.Pubmed PMID: 2439677.

[4]. Jathal B, Trivedi A, Bhavsar N. Use of fibrin glue in periodontal flap surgery. J Indian Soc Periodontol. 2008 Jan;12(1):21

[5]. Manimegalai AG. A comparative study on the efficacy of a commercial fibrin adhesive (Tisseel) vis-à-vis silk suture on wound closure following periodontal surgical procedures. J Indian Soc Periodontol. 2010 Oct;14(4):231-5. Pubmed PMID: 21731248.

[6]. Warrer K, Karring T. Effect of Tisseel ${ }^{\circ}$ on healing after periodontal flap surgery. J. Clin. Periodontol. 1992 Aug;19(7):449-54.

[7]. Romanos GE, Strub JR. Effect of Tissucol on connective tissue matrix during wound healing: an immunohistochemical study in rat skin. J Biomed Mater Res. 1998 Mar 5;39(3):462-8.Pubmed PMID: 9468057.

[8]. Eskan MA, Greenwell H. Theoretical and clinical considerations for autologous blood preparations: Platelet-rich plasma, fibrin sealants, and plasmarich growth factors. Clin Adv Periodontics. 2011 Aug;1(2):142-53.

[9]. Barbosa MD, Stipp AC, Passanezi E, Greghi SL. Fibrin adhesive derived from snake venom in periodontal surgery: histological analysis. J Appl Oral Sci. 2008 Sep-Oct;16(5):310-5.Pubmed PMID: 19089226.

[10]. Le Guéhennec L, Layrolle P, Daculsi G. A review of bioceramics and fibrin sealant. Eur Cell Mater. 2004 Sep 13;8:1-11.Pubmed PMID: 15494929.

[11]. Thompson DF, Davis TW. The addition of antibiotics to fibrin glue. South. Med. J. 1997 Jul 1;90(7):681-4.

[12]. Spotnitz WD. Fibrin sealant in the United States: clinical use at the University of Virginia. Thromb Haemost. 1995 Jul;74(1):482-5.Pubmed PMID: 8578510 .

[13]. Ohazama A, Isatsu K, Hatayama J, Okamatsu Y, Tachikawa T, Hasegawa K. Periodontal tissue regeneration using fibrin tissue adhesive material in vitro and in vivo. Periodontal Clin Investig. 1996 Spring;18(1):26-38.Pubmed PMID: 9116461.

[14]. Alston SM, Solen KA, Broderick AH, Sukavaneshvar S, Mohammad SF. New method to prepare autologous fibrin glue on demand. Transl Res. 2007 Apr;149(4):187-95.Pubmed PMID: 17383592.

[15]. Hartman AR, Galanakis DK, Honig MP, Seifert FC, Anagnostopoulos CE. Autologous whole plasma fibrin gel: intraoperative procurement. Arch Surg. 1992 Mar 1;127(3):357-9.

[16]. Polson AM, Proye MP. Fibrin linkage: a precursor for new attachment. J Periodontol. 1983 Mar;54(3):141-7.Pubmed PMID: 6573470.

[17]. Becker W, Goldstein M, Becker BE, Sennerby L. Minimally invasive flapless implant surgery: a prospective multicenter study. Clin Implant Dent Relat Res. 2005 Jun;7:s21-7.

[18]. Dave BP, Sathyanarayana S. Periodontal flap closure using a simplified autologous fibrin glue preparation: A report of two successful cases. J Indian Soc Periodontol. 2020 Nov-Dec;24(6):579-582.Pubmed PMID: 33424178.

[19]. Landry RG. Effectiveness of Benzydamine HC1 in the Treatment of Periodontal Post-surgical Patients: Faculty of Dentistry. University of Toronto. 1985. 